FERMILAB-Conf-02/209-T

hep-ph/0209008

\title{
Lifetimes of heavy hadrons beyond leading logarithms*
}

\author{
Ulrich Nierste \\ Fermi National Accelerator Laboratory \\ Batavia, IL60510 - 500, USA. ${ }^{\dagger}$ \\ E-mail: nierste@fnal.gov
}

\begin{abstract}
The lifetime splitting between the $B^{+}$and $B_{d}^{0}$ mesons has recently been calculated in the next-to-leading order of QCD. These corrections are necessary for a reliable theoretical prediction, in particular for the meaningful use of hadronic matrix elements computed with lattice QCD. Using results from quenched lattice QCD we find $\tau\left(B^{+}\right) / \tau\left(B_{d}^{0}\right)=1.053 \pm 0.016 \pm 0.017$, where the uncertainties from unquenching and $1 / m_{b}$ corrections are not included. The lifetime difference of heavy baryons $\Xi_{b}^{0}$ and $\Xi_{b}^{-}$is also discussed.
\end{abstract}

\footnotetext{
*Invited talk at Continuous Advances in QCD 2002/ARKADYFEST (honoring the 60th birthday of Prof. Arkady Vainshtein), 17-23 May 2002, Minneapolis, Minnesota, USA.

${ }^{\dagger}$ Fermilab is operated by URA under DOE contract No. DE-AC02-76CH03000.
} 


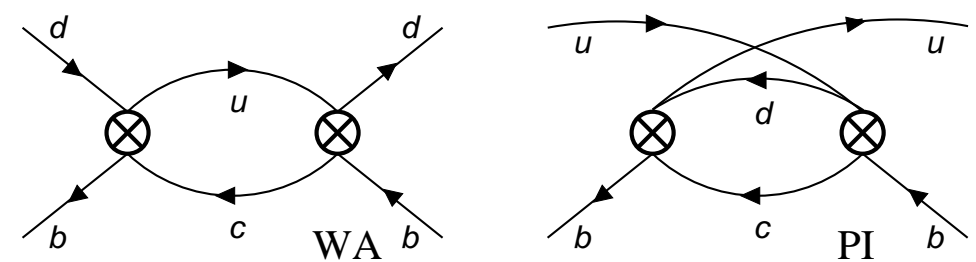

Figure 1: Weak annihilation (WA) and Pauli interference (PI) diagrams in the leading order of QCD. They contribute to $\Gamma\left(B_{d}^{0}\right)$ and $\Gamma\left(B^{+}\right)$, respectively. The crosses represent $|\Delta B|=1$ operators, which are generated by the exchange of $W$ bosons. CKM-suppressed contributions are not shown.

\section{Introduction}

In my talk I present work done in collaboration with Martin Beneke, Gerhard Buchalla, Christoph Greub and Alexander Lenz [1].

Twenty years ago the hosts of this conference showed that inclusive decay rates of hadrons containg a heavy quark can be computed from first principles of QCD. The Heavy Quark Expansion (HQE) technique [2] exploits the heaviness of the bottom (or charm) quark compared to the fundamental QCD scale $\Lambda_{Q C D}$. In order to study the lifetime of some $b$-flavored hadron $H$ containing a single heavy quark one needs to compute its total decay rate $\Gamma\left(H_{b}\right)$. Now the HQE is an operator product expansion (OPE) expressing $\Gamma\left(H_{b}\right)$ in terms of matrix elements of local $\Delta B=0$ ( $B$ denotes the bottom number) operators, leading to an expansion of $\Gamma\left(H_{b}\right)$ in terms of $\Lambda_{Q C D} / m_{b}$. In the leading order of $\Lambda_{Q C D} / m_{b}$ the decay rate of $H_{b}$ equals the decay rate of a free $b$-quark, unaffected by the light degrees of freedom of $H_{b}$. Consequently, the lifetimes of all $b$-flavored hadrons are the same at this order. The dominant source of lifetime differences are weak interaction effects between the $b$-quark and the light valence quark. They are depicted in Fig. 1 for the case of the $B^{+}-B_{d}^{0}$ lifetime difference. The relative size of these weak non-spectator effects to the leading free-quark decay is of order $16 \pi^{2}\left(\Lambda_{Q C D} / m_{b}\right)^{3}=\mathcal{O}(5-10 \%)$. The measurement of lifetime differences among different $b$-flavored hadrons therefore tests the HQE formalism at the third order in the expansion parameter.

The optical theorem relates the total decay rate $\Gamma\left(H_{b}\right)$ to the self-energy of $H_{b}$ :

$$
\Gamma\left(H_{b}\right)=\frac{1}{2 M_{H_{b}}}\left\langle H_{b}|\mathcal{T}| H_{b}\right\rangle .
$$

Here we have introduced the transition operator:

$$
\mathcal{T}=\operatorname{Im} i \int d^{4} x T[H(x) H(0)]
$$

with the effective $|\Delta B|=1$ Hamiltonian $H$ describing the $W$-mediated decay of the $b$ quark. The HQE amounts to an OPE applied to $\mathcal{T}$ which effectively integrates out the hard loop momenta (corresponding to the momenta of the final state quarks). We 
decompose the result as

$$
\begin{aligned}
\mathcal{T} & =\left[\mathcal{T}_{0}+\mathcal{T}_{2}+\mathcal{T}_{3}\right]\left[1+\mathcal{O}\left(1 / m_{b}^{4}\right)\right] \\
\mathcal{T}_{3} & =\mathcal{T}^{u}+\mathcal{T}^{d}+\mathcal{T}_{\text {sing }}
\end{aligned}
$$

Here $\mathcal{T}_{n}$ denotes the portion of $\mathcal{T}$ which is suppressed by a factor of $1 / m_{b}^{n}$ with respect to $\mathcal{T}_{0}$ describing the free quark decay. The contributions to $\mathcal{T}_{3}$ from the weak interaction with the valence quark read

$$
\begin{aligned}
& \mathcal{T}^{u}=\frac{G_{F}^{2} m_{b}^{2}\left|V_{c b}\right|^{2}}{6 \pi}\left[\left|V_{u d}\right|^{2}\left(F^{u} Q^{d}+F_{S}^{u} Q_{S}^{d}+G^{u} T^{d}+G_{S}^{u} T_{S}^{d}\right)\right. \\
& \left.+\left|V_{c d}\right|^{2}\left(F^{c} Q^{d}+F_{S}^{c} Q_{S}^{d}+G^{c} T^{d}+G_{S}^{c} T_{S}^{d}\right)\right] \\
& +(d \rightarrow s) \\
& \mathcal{T}^{d}=\frac{G_{F}^{2} m_{b}^{2}\left|V_{c b}\right|^{2}}{6 \pi}\left[F^{d} Q^{u}+F_{S}^{d} Q_{S}^{u}+G^{d} T^{u}+G_{S}^{d} T_{S}^{u}\right] .
\end{aligned}
$$

Here $G_{F}$ is the Fermi constant, $m_{b}$ is the bottom mass and the $V_{i j}$ 's are elements of the Cabibbo-Kobayashi-Maskawa (CKM) matrix. The superscript $q$ of the coefficients $F^{q}$, $F_{S}^{q}, G^{q}, G_{S}^{q}$ refers to the $c q$ intermediate state. The leading contributions to $\mathcal{T}^{u}$ and $\mathcal{T}^{d}$ are obtained from the left and right diagram in Fig. 1, respectively. They involve the local dimension- $6, \Delta B=0$ operators

$$
\begin{aligned}
Q^{q} & =\bar{b} \gamma_{\mu}\left(1-\gamma_{5}\right) q \bar{q} \gamma^{\mu}\left(1-\gamma_{5}\right) b, \\
Q_{S}^{q} & =\bar{b}\left(1-\gamma_{5}\right) q \bar{q}\left(1+\gamma_{5}\right) b, \\
T^{q} & =\bar{b} \gamma_{\mu}\left(1-\gamma_{5}\right) T^{a} q \bar{q} \gamma^{\mu}\left(1-\gamma_{5}\right) T^{a} b, \\
T_{S}^{q} & =\bar{b}\left(1-\gamma_{5}\right) T^{a} q \bar{q}\left(1+\gamma_{5}\right) T^{a} b,
\end{aligned}
$$

where $T^{a}$ is the generator of color SU(3). The Wilson coefficients $F^{u} \ldots G_{S}^{d}$ contain the physics from scales above $m_{b}$ and are computed in perturbation theory. The remain$\operatorname{der} \mathcal{T}_{\text {sing }}$ in (3) involves additional dimension-6 operators, which are $S U(3)_{F}$ singlets and do not contribute to the lifetime splitting within the $\left(B^{+}, B_{d}^{0}\right)$ and $\left(\Xi_{b}^{0}, \Xi_{b}^{-}\right)$isodoublets. In order to predict the widths $\Gamma\left(B_{d}^{0}\right)$ and $\Gamma\left(B^{+}\right)$one needs to compute the hadronic matrix elements of the operators in (5). After using the isospin relation $\left\langle B_{d}^{0}\left|Q^{d, u}\right| B_{d}^{0}\right\rangle=\left\langle B^{+}\left|Q^{u, d}\right| B^{+}\right\rangle$the matrix elements will enter $\Gamma\left(B_{d}^{0}\right)-\Gamma\left(B^{+}\right)$in isospin-breaking combinations, which are conventionally parametrized as [3, 4 ]

$$
\begin{aligned}
& \left\langle B^{+}\left|\left(Q^{u}-Q^{d}\right)\right| B^{+}\right\rangle=f_{B}^{2} M_{B}^{2} B_{1}, \quad\left\langle B^{+}\left|\left(Q_{S}^{u}-Q_{S}^{d}\right)\right| B^{+}\right\rangle=f_{B}^{2} M_{B}^{2} B_{2}, \\
& \left\langle B^{+}\left|\left(T^{u}-T^{d}\right)\right| B^{+}\right\rangle=f_{B}^{2} M_{B}^{2} \epsilon_{1}, \quad\left\langle B^{+}\left|\left(T_{S}^{u}-T_{S}^{d}\right)\right| B^{+}\right\rangle=f_{B}^{2} M_{B}^{2} \epsilon_{2} .
\end{aligned}
$$

Here $f_{B}$ and $M_{B}$ are decay constant and mass of the $B$ meson, respectively. In the vacuum saturation approximation (VSA) one has $B_{1}=1, B_{2}=1+\mathcal{O}\left(\alpha_{s}\left(m_{b}\right), \Lambda_{Q C D} / m_{b}\right)$ and $\epsilon_{1,2}=0$. Corrections to the VSA results are of order $1 / N_{c}$, where $N_{c}=3$ is the number of colors. 
We now find from (11) and (4):

$$
\Gamma\left(B_{d}^{0}\right)-\Gamma\left(B^{+}\right)=\frac{G_{F}^{2} m_{b}^{2}\left|V_{c b}\right|^{2}}{12 \pi} f_{B}^{2} M_{B}\left(\left|V_{u d}\right|^{2} \vec{F}^{u}+\left|V_{c d}\right|^{2} \vec{F}^{c}-\vec{F}^{d}\right) \cdot \vec{B} .
$$

Here we have introduced the shorthand notation

$$
\vec{F}^{q}(z)=\left(\begin{array}{c}
F^{q}(z) \\
F_{S}^{q}(z) \\
G^{q}(z) \\
G_{S}^{q}(z)
\end{array}\right), \quad \vec{B}=\left(\begin{array}{c}
B_{1} \\
B_{2} \\
\epsilon_{1} \\
\epsilon_{2}
\end{array}\right) \quad \text { for } q=d, u, c .
$$

Since the hard loops involve the charm quark, the coefficient $\vec{F}^{q}$ depends on the ratio $z=m_{c}^{2} / m_{b}^{2}$. The minimal way to include QCD effects is the leading logarithmic approximation, which includes corrections of order $\alpha_{s}^{n} \ln ^{n}\left(m_{b} / M_{W}\right), n=0,1, \ldots$ in $\vec{F}^{q}$ in $(7)$. The corresponding leading order (LO) calculation of the width difference in (7) involves the diagrams in Fig. 1 [2, 3]. Yet LO results are too crude for a precise calculation of lifetime differences. The heavy-quark masses in (7) cannot be defined in a proper way and one faces a large dependence on unphysical renormalization scales. Furthermore, results for $B_{1,2}$ and $\epsilon_{1,2}$ from lattice gauge theory cannot be matched to the continuum theory in a meaningful way at LO. Finally, as pointed out in [ [ $]$ ], at LO the coefficients $F, F_{S}$ in (77) are anomalously small. They multiply the large matrix elements parametrized by $B_{1,2}$, while the larger coefficients $G, G_{S}$ come with the small hadronic parameters $\epsilon_{1,2}$, rendering the LO prediction highly unstable. To cure these problems one must include the next-to-leading-order (NLO) QCD corrections of order $\alpha_{s}^{n+1} \ln ^{n}\left(m_{b} / M_{W}\right)$.

The first calculation of a lifetime difference beyond the LO was performed for the $B_{s}^{0}-B_{d}^{0}$ lifetime difference [5], where $\mathcal{O}\left(\alpha_{s}\right)$ corrections were calculated in the $\mathrm{SU}(3)_{\mathrm{F}}$ limit neglecting certain terms of order $z$. In this limit only a few penguin effects play a role. A complete NLO computation has been carried out for the lifetime difference between the two mass eigenstates of the $B_{s}^{0}$ meson in [6]. In particular the correct treatment of infrared effects, which appear at intermediate steps of the calculation, has been worked out in [6]. The recent computation in [1] is conceptually similar to the one in [6], except that the considered transition is $\Delta B=0$ rather than $\Delta B=2$ and the quark masses in the final state are different. The NLO calculation of $\Gamma\left(B_{d}^{0}\right)-\Gamma\left(B^{+}\right)$ involves the diagrams of Fig. [2. In [ [ 4 the NLO corrections to $\Gamma\left(B_{d}^{0}\right)-\Gamma\left(B^{+}\right)$have been calculated for the limiting case $z=0$. The corrections to this limit are of order $z \ln z$ or roughly $20 \%$. The first NLO calculation with the complete $z$ dependence was presented in [1] and subsequently confirmed in [7].

\section{Lifetime differences at next-to-leading order}

The analytic expressions for the Wilson coefficients $F_{i j}^{u,(1)}-F_{i j}^{d,(1)} \ldots G_{S, i j}^{u,(1)}-G_{S, i j}^{d,(1)}$ are cumbersome functions of $z$ involving dilogarithms. They depend on the renormalization scheme chosen for the $\Delta B=0$ operators in $(5)$ and also on the renormalization scale $\mu_{0}=\mathcal{O}\left(m_{b}\right)$ at which these operators are defined. These dependences properly cancel between $\vec{F}^{q}$ and $\vec{B}$ in physical observables like $(7)$. When our results for 


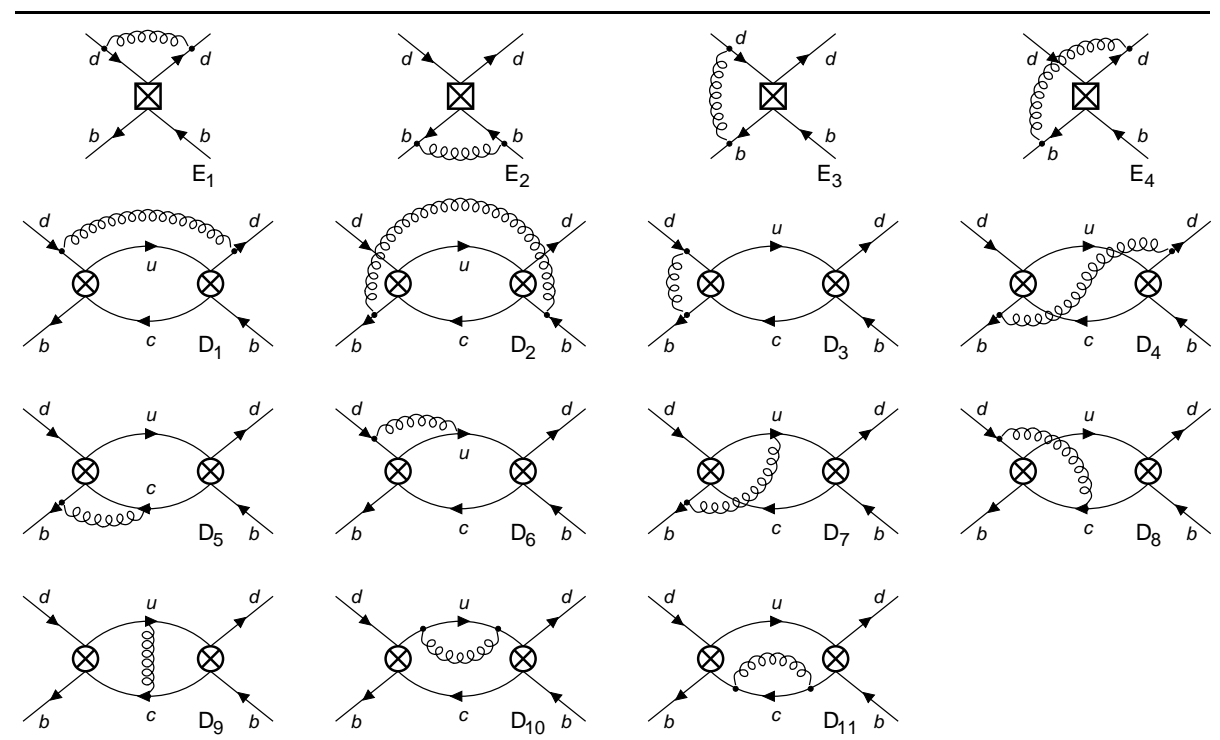

Figure 2: WA contributions in the next-to-leading order of QCD. The PI diagrams are obtained by interchanging $u$ and $d$ and reversing the fermion flow of the $u$ and $d$ lines. The first line shows the radiative corrections to $\Delta B=0$ operators, which are necessary for the proper infrared factorization. Not displayed are the diagrams $\mathrm{E}_{3}^{\prime}, \mathrm{E}_{4}^{\prime}$ and $\mathrm{D}_{3-8}^{\prime}$ which are obtained from the corresponding unprimed diagrams by left-right reflection and the reverse of the fermion flow.

$F_{i j}^{u,(1)}-F_{i j}^{d,(1)} \ldots G_{S, i j}^{u,(1)}-G_{S, i j}^{d,(1)}$ are combined with some non-perturbative computation of $B_{1}, \ldots \epsilon_{2}$, one has to make sure that the numerical values of these hadronic parameters correspond to the same renormalization scheme. Our scheme is defined by the use of dimensional regularization with $\overline{\mathrm{MS}}$ [8] subtraction, an anticommuting $\gamma_{5}$ and a choice of evanescent operators preserving Fierz invariance at the loop level [9]. Choosing further $\mu_{0}=m_{b}$ the desired lifetime ratio can be compactly written as

$$
\begin{aligned}
\frac{\tau\left(B^{+}\right)}{\tau\left(B_{d}^{0}\right)}-1=\tau\left(B^{+}\right)\left[\Gamma\left(B_{d}^{0}\right)-\Gamma\left(B^{+}\right)\right] \\
=0.0325\left(\frac{\left|V_{c b}\right|}{0.04}\right)^{2}\left(\frac{m_{b}}{4.8 \mathrm{GeV}}\right)^{2}\left(\frac{f_{B}}{200 \mathrm{MeV}}\right)^{2} \times \\
\quad\left[(1.0 \pm 0.2) B_{1}+(0.1 \pm 0.1) B_{2}-(18.4 \pm 0.9) \epsilon_{1}+(4.0 \pm 0.2) \epsilon_{2}\right] .
\end{aligned}
$$

Here $\tau\left(B^{+}\right)=1.653 \mathrm{ps}$ has been used in the overall factor.

The hadronic parameters have been computed in [10] with quenched lattice QCD using the same renormalization scheme as in the present paper. They read

$$
\left(B_{1}, B_{2}, \epsilon_{1}, \epsilon_{2}\right)=(1.10 \pm 0.20,0.79 \pm 0.10,-0.02 \pm 0.02,0.03 \pm 0.01) .
$$

Inserting $\left|V_{c b}\right|=0.040 \pm 0.0016$ from a CLEO analysis of inclusive semileptonic $B$ 
decays [13], the world average $f_{B}=(200 \pm 30) \mathrm{MeV}$ from lattice calculations [14] and $m_{b}=4.8 \pm 0.1 \mathrm{GeV}$ for the one-loop bottom pole mass into (9), our NLO prediction reads

$$
\frac{\tau\left(B^{+}\right)}{\tau\left(B_{d}^{0}\right)}=1.053 \pm 0.016 \pm 0.017
$$

compared to

$$
\left[\frac{\tau\left(B^{+}\right)}{\tau\left(B_{d}^{0}\right)}\right]_{\mathrm{LO}}=1.041 \pm 0.040 \pm 0.013
$$

Here the first error is due to the errors on the coefficients and the hadronic parameters (10), and the second error is the overall normalization uncertainty due to $m_{b},\left|V_{c b}\right|$ and $f_{B}$ in (9). The Wilson coefficients also depend on the renormalization scale $\mu_{1}$ at which the $\Delta B=1$ operators entering the diagrams in Figs. 1 and 2 are defined. This dependence stems from the truncation of the perturbation series and diminishes orderby-order in $\alpha_{s}$. The dependence on $\mu_{1}$ is the dominant uncertainty of the LO prediction of the lifetime ratio. In Fig. 3 the $\mu_{1}$-dependence of the LO and NLO predictions for $\tau\left(B^{+}\right) / \tau\left(B_{d}^{0}\right)-1$ is shown. The substantial reduction of scale dependence at NLO leads to the improvement in the NLO vs. LO results in $(11),(12)$. Note that the NLO calculation has firmly established that $\tau\left(B^{+}\right)>\tau\left(B_{d}^{0}\right)$, a conclusion which could not be drawn from the old LO result. The result in (11) is compatible with recent measurement from the B factories [11, 12]:

$$
\frac{\tau\left(B^{+}\right)}{\tau\left(B_{d}^{0}\right)}= \begin{cases}1.082 \pm 0.026 \pm 0.012 & (B A B A R) \\ 1.091 \pm 0.023 \pm 0.014 & \text { (BELLE) }\end{cases}
$$

The calculated Wilson coefficients can also be used to predict the lifetime splitting within the iso-doublet $\left(\Xi_{b}^{0} \sim b u s, \Xi_{b}^{-} \sim b d s\right)$ with NLO precision. The corresponding LO diagrams are shown in Fig. 4 . Note that the role of $\mathcal{T}^{u}$ and $\mathcal{T}^{d}$ is interchanged compared to the meson case with $\mathcal{T}^{u}$ describing the Pauli interference effect. The lifetime difference between $\Lambda_{b} \sim b u d$ and $\Xi_{b}^{0}$ is expected to be small, as in the case of $B_{s}^{0}$ and $B_{d}^{0}$, because it mainly stems from the small U-spin breaking effects in the matrix elements appearing at order $1 / m_{b}^{2}$.

For $\Xi_{b}$ 's the weak decay of the valence $s$-quark could be relevant: the decays $\Xi_{b}^{-} \rightarrow$ $\Lambda_{b} \pi^{-}, \Xi_{b}^{-} \rightarrow \Lambda_{b} e^{-} \bar{\nu}_{e}$ and $\Xi_{b}^{0} \rightarrow \Lambda_{b} \pi^{0}$ are triggered by $s \rightarrow u$ transitions and could affect the total rates at the $\mathcal{O}(1 \%)$ level [15]. Once the lifetime measurements reach this accuracy, one should correct for this effect. To this end we define

$$
\begin{array}{r}
\bar{\Gamma}\left(\Xi_{b}\right) \equiv \Gamma\left(\Xi_{b}\right)-\Gamma\left(\Xi_{b} \rightarrow \Lambda_{b} X\right)=\frac{1-B\left(\Xi_{b} \rightarrow \Lambda_{b} X\right)}{\tau\left(\Xi_{b}\right)} \equiv \frac{1}{\bar{\tau}\left(\Xi_{b}\right)} \\
\text { for } \Xi_{b}=\Xi_{b}^{0}, \Xi_{b}^{-},
\end{array}
$$

where $B\left(\Xi_{b} \rightarrow \Lambda_{b} X\right)$ is the branching ratio of the above-mentioned decay modes. Thus $\bar{\Gamma}\left(\Xi_{b}\right)$ is the contribution from $b \rightarrow c$ transitions to the total decay rate. In contrast to the $B$ meson system, the matrix elements of the four operators in (5) are not independent at the considered order in $\Lambda_{Q C D} / m_{b}$. Since the light degrees of freedom 


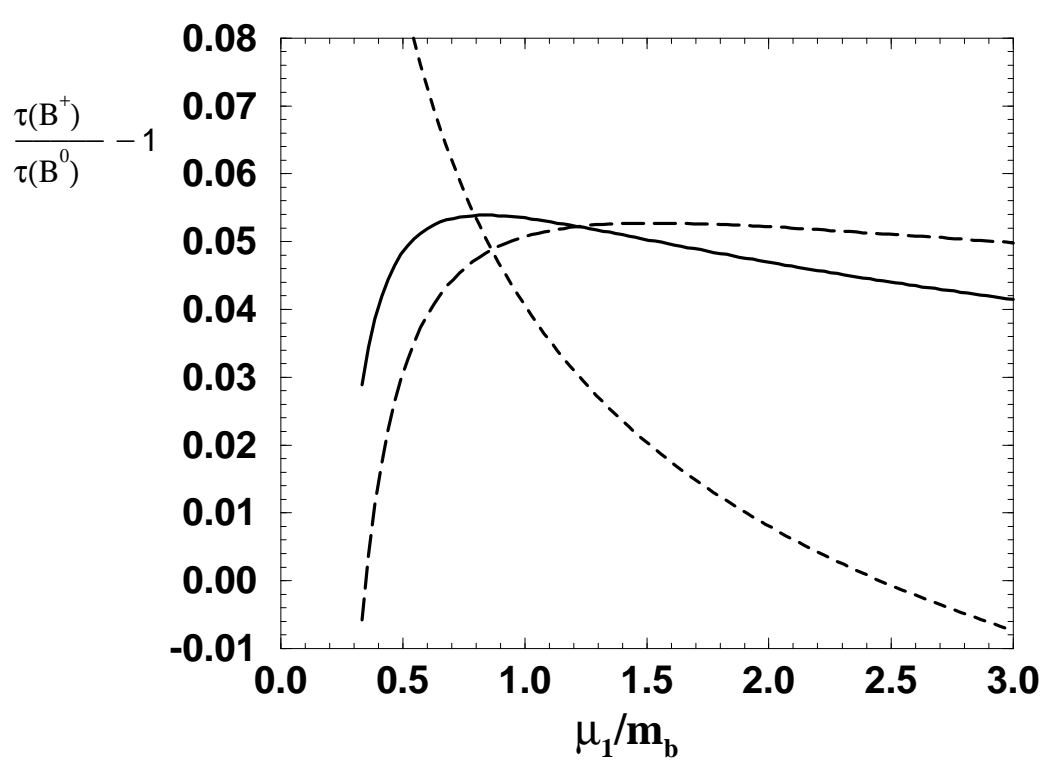

Figure 3: Dependence of $\tau\left(B^{+}\right) / \tau\left(B_{d}^{0}\right)-1$ on $\mu_{1} / m_{b}$ for the central values of the input parameters and $\mu_{0}=m_{b}$. The solid (short-dashed) line shows the NLO (LO) result. The long-dashed line shows the NLO result in the approximation of [ $₫$ ], i.e. $z$ is set to zero in the NLO corrections.

are in a spin-0 state, the matrix elements $\left\langle\Xi_{b}\left|2 Q_{S}^{q}+Q^{q}\right| \Xi_{b}\right\rangle$ and $\left\langle\Xi_{b}\left|2 T_{S}^{q}+T^{q}\right| \Xi_{b}\right\rangle$ are power-suppressed compared to those in (14) (see e.g. [2, 3]). This, however, is not true in all renormalization schemes, in the $\overline{\mathrm{MS}}$ scheme used by us $2 Q_{S}^{q}+Q^{q}$ and $2 T_{S}^{q}+T^{q}$ receive short-distance corrections, because hard gluons can resolve the heavy $b$-quark mass. A priori one can choose the renormalization of e.g. $Q_{S}^{q}$ independently from $Q^{q}$, so that $\left\langle\Xi_{b}\left|2 Q_{S}^{q}+Q^{q}\right| \Xi_{b}\right\rangle=\mathcal{O}\left(\Lambda_{Q C D} / m_{b}\right)$ can only hold in certain renormalization schemes. This is also the case, if the operators are defined in heavy quark effective theory (HQET) rather than in full QCD. After properly taking into account these shortdistance corrections, one can express the desired lifetime ratio solely in terms of two hadronic parameters defined as

$$
\begin{aligned}
\left\langle\Xi_{b}^{0}\left|\left(Q^{u}-Q^{d}\right)\left(\mu_{0}\right)\right| \Xi_{b}^{0}\right\rangle & =f_{B}^{2} M_{B} M_{\Xi_{b}} L_{1}^{\Xi_{b}}\left(\mu_{0}\right), \\
\left\langle\Xi_{b}^{0}\left|\left(T^{u}-T^{d}\right)\left(\mu_{0}\right)\right| \Xi_{b}^{0}\right\rangle & =f_{B}^{2} M_{B} M_{\Xi_{b}} L_{2}^{\Xi_{b}}\left(\mu_{0}\right) .
\end{aligned}
$$



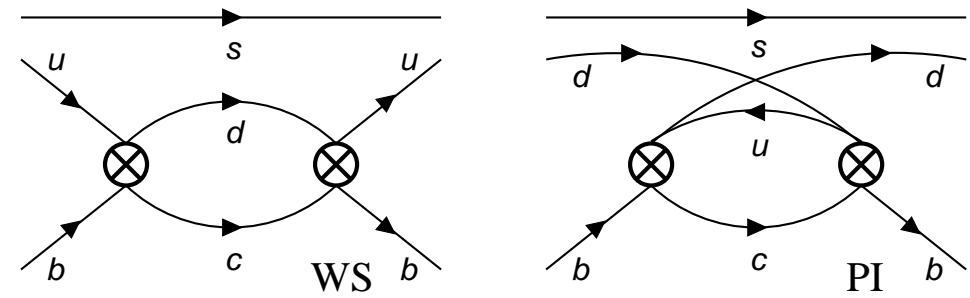

Figure 4: Weak scattering (WS) and PI diagrams for $\Xi_{b}$ baryons in the leading order of QCD. They contribute to $\Gamma\left(\Xi_{b}^{0}\right)$ and $\Gamma\left(\Xi_{b}^{-}\right)$, respectively. CKM-suppressed contributions are not shown.

Then one finds

$$
\begin{aligned}
\frac{\bar{\tau}\left(\Xi_{b}^{0}\right)}{\bar{\tau}\left(\Xi_{b}^{-}\right)}-1= & \bar{\tau}\left(\Xi_{b}^{0}\right)\left[\Gamma\left(\Xi_{b}^{-}\right)-\Gamma\left(\Xi_{b}^{0}\right)\right] \\
= & 0.59\left(\frac{\left|V_{c b}\right|}{0.04}\right)^{2}\left(\frac{m_{b}}{4.8 \mathrm{GeV}}\right)^{2}\left(\frac{f_{B}}{200 \mathrm{MeV}}\right)^{2} \frac{\bar{\tau}\left(\Xi_{b}^{0}\right)}{1.5 \mathrm{ps}} \times \\
& \quad\left[(0.04 \pm 0.01) L_{1}-(1.00 \pm 0.04) L_{2}\right],
\end{aligned}
$$

with $L_{i}=L_{i}^{\Xi_{b}}\left(\mu_{0}=m_{b}\right)$. For the baryon case there is no reason to expect the coloroctet matrix element to be much smaller than the color-singlet ones, so that the term with $L_{2}$ will dominate the result. The hadronic parameters $L_{1,2}$ have been analyzed in an exploratory study of lattice HQET [16] for $\Lambda_{b}$ baryons. Up to $\mathrm{SU}(3)_{\mathrm{F}}$ corrections, which are irrelevant in view of the other uncertainties, $L_{i}^{\Xi_{b}}$ and $L_{i}^{\Lambda_{b}}$ are equal.

\section{Conclusions}

Twenty years ago the ITEP group has developed the Heavy Quark Expansion, which allows to study inclusive decay rates of heavy hadrons in a model-free, QCD-based framework [2]. The HQE expresses these decay rates as a series in both $\Lambda_{Q C D} / m_{b}$ and $\alpha_{s}\left(m_{b}\right)$. With the advent of precision measurements of lifetimes of $b$-flavored hadrons at the $\mathrm{B}$ factories and the Tevatron correspondingly precise theory predictions are desirable. This requires the calculation of higher-order terms in the HQE. The inclusion of the $\alpha_{s}$ corrections presented in this talk is in particular mandatory for any meaningful use of hadronic matrix elements computed in lattice gauge theory. The calculated QCD corrections to the WA and PI diagrams in Figs. 11,2 allow to study the lifetime splitting within the $\left(B^{+}, B_{d}^{0}\right)$ and $\left(\Xi_{b}^{0}, \Xi_{b}^{-}\right)$iso-doublets with NLO accuracy. It is gratifying that these corrections have been independently calulated by two groups finding agreement in their analytic expressions for the Wilson coefficients [1, 7 ].

Current lattice calculations, which are still in a relatively early stage in this case, yield, when combined with our calculations, $\tau\left(B^{+}\right) / \tau\left(B_{d}^{0}\right)=1.053 \pm 0.016 \pm 0.017$ [see [11]. The effects of unquenching and $1 / m_{b}$ corrections are not included in the 
error estimate, but the unquenching effects can well be sizable. A substantial improvement of the NLO calculation is the large reduction of perturbative uncertainty reflected in the scale dependence stemming from the $\Delta B=1$ operators. This scale dependence had been found to be very large at leading order, preventing even an unambiguous prediction of the sign of $\tau\left(B^{+}\right) / \tau\left(B_{d}^{0}\right)-1$ up to now [3].

At present the experimentally measured $\Lambda_{b}$ lifetime falls short of $\tau\left(B_{d}^{0}\right)$ by roughly $20 \%$ [17], which has raised concerns about the applicability of the HQE to baryons. Unfortunately this interesting topic cannot yet be addressed at the NLO level for two reasons: First, $\tau\left(\Lambda_{b}\right) / \tau\left(B_{d}^{0}\right)$ receives contributions from the yet uncalculated $\mathrm{SU}(3)_{\mathrm{F}}{ }^{-}$ singlet portion $\mathcal{T}_{\text {sing }}$ of the transition operator in (3). Second, the hadronic matrix elements entering $\tau\left(\Lambda_{b}\right) / \tau\left(B_{d}^{0}\right)$ involve penguin (also called 'eye') contractions of the operators in (5), which are difficult to compute. These penguin contractions are contributions to the matrix elements in which the light $q$ and $\bar{q}$ quark fields of the operator are contracted with each other, not with the hadron's valence quarks.

\section{Acknowledgments}

I thank the organizers for the invitation to this wonderful Arkadyfest workshop. I have enjoyed stimulating discussions with many participants and look forward to future Arkadyfests, possibly on the occasions of Arkady's 70th, 80th, 90th and 100th birthdays! I am grateful to Martin Gorbahn for proofreading the manuscript.

\section{References}

[1] M. Beneke, G. Buchalla, C. Greub, A. Lenz and U. Nierste, Nucl. Phys. B 639 (2002) 389 [arXiv:hep-ph/0202106].

[2] M. A. Shifman and M. B. Voloshin, in: Heavy Quarks ed. V. A. Khoze and M. A. Shifman, Sov. Phys. Usp. 26 (1983) 387; M. A. Shifman and M. B. Voloshin, Sov. J. Nucl. Phys. 41 (1985) 120 [Yad. Fiz. 41 (1985) 187]; M. A. Shifman and M. B. Voloshin, Sov. Phys. JETP 64 (1986) 698 [Zh. Eksp. Teor. Fiz. 91 (1986) 1180]; I. I. Bigi, N. G. Uraltsev and A. I. Vainshtein, Phys. Lett. B 293 (1992) 430 [Erratum-ibid. B 297 (1992) 477]. For a recent review see: M. Voloshin, in: $B$ physics at the Tevatron: Run-II and Beyond, Chapter 8, [hep-ph/0201071].

[3] M. Neubert and C. T. Sachrajda, Nucl. Phys. B 483 (1997) 339. M. Beneke, G. Buchalla and I. Dunietz, Phys. Rev. D 54 (1996) 4419.

[4] M. Ciuchini, E. Franco, V. Lubicz and F. Mescia, [hep-ph/0110375].

[5] Y. Y. Keum and U. Nierste, Phys. Rev. D 57 (1998) 4282.

[6] M. Beneke, G. Buchalla, C. Greub, A. Lenz and U. Nierste, Phys. Lett. B 459 (1999) 631.

[7] E. Franco, V. Lubicz, F. Mescia and C. Tarantino, Nucl. Phys. B 633 (2002) 212 [arXiv:hep-ph/0203089]. 
[8] W. A. Bardeen, A. J. Buras, D. W. Duke and T. Muta, Phys. Rev. D 18 (1978) 3998.

[9] S. Herrlich and U. Nierste, Nucl. Phys. B 455 (1995) 39.

[10] D. Becirevic, [hep-ph/0110124].

[11] B. Aubert et al. [BABAR Collaboration], Phys. Rev. Lett. 87 (2001) 201803.

[12] K. Abe [Belle Collaboration], [hep-ex/0202009].

[13] D. Cassel [CLEO coll.], talk at Lepton Photon 01, 23-28 Jul 2001, Rome, Italy.

[14] S. Ryan, hep-lat/0111010].

[15] M. B. Voloshin, Phys. Lett. B 476 (2000) 297.

[16] M. Di Pierro, C. T. Sachrajda and C. Michael [UKQCD collaboration], Phys. Lett. B 468 (1999) 143.

[17] D. E. Groom et al. [Particle Data Group Collaboration], Eur. Phys. J. C 15 (2000) 1 ; updated at $h t t p: / / p d g . l b l . g o v$. 